\title{
CRALBP supports the mammalian retinal visual cycle and cone vision
}

\author{
Yunlu Xue, ${ }^{1,2}$ Susan Q. Shen, ${ }^{2,3}$ Jonathan Jui, ${ }^{4}$ Alan C. Rupp, ${ }^{5}$ Leah C. Byrne, ${ }^{4}$ Samer Hattar, ${ }^{5}$ John G. Flannery, ${ }^{4}$ \\ Joseph C. Corbo, ${ }^{3}$ and Vladimir J. Kefalov ${ }^{1}$ \\ 'Department of Ophthalmology and Visual Sciences, ${ }^{2}$ Graduate Program in Division of Biological and Biomedical Sciences, and ${ }^{3}$ Department of Pathology and Immunology, \\ Washington University School of Medicine, St. Louis, Missouri, USA. ${ }^{4}$ Helen Wills Neuroscience Institute, University of California, Berkeley, California, USA. \\ ${ }^{5}$ Department of Biology, Johns Hopkins University, Baltimore, Maryland, USA.
}

\begin{abstract}
Mutations in the cellular retinaldehyde-binding protein (CRALBP, encoded by RLBP1) can lead to severe cone photoreceptormediated vision loss in patients. It is not known how CRALBP supports cone function or how altered CRALBP leads to cone dysfunction. Here, we determined that deletion of RIbp1 in mice impairs the retinal visual cycle. Mice lacking CRALBP exhibited M-opsin mislocalization, M-cone loss, and impaired cone-driven visual behavior and light responses. Additionally, M-cone dark adaptation was largely suppressed in CRALBP-deficient animals. While rearing CRALBP-deficient mice in the dark prevented the deterioration of cone function, it did not rescue cone dark adaptation. Adeno-associated virus-mediated restoration of CRALBP expression specifically in Müller cells, but not retinal pigment epithelial (RPE) cells, rescued the retinal visual cycle and $\mathrm{M}$-cone sensitivity in knockout mice. Our results identify Müller cell CRALBP as a key component of the retinal visual cycle and demonstrate that this pathway is important for maintaining normal cone-driven vision and accelerating cone dark adaptation.
\end{abstract}

\section{Introduction}

Photoactivation of a visual pigment molecule in vertebrate rod and cone photoreceptors rapidly triggers a light response and concomitantly renders the activated pigment unable to detect a subsequent photon of light. Regeneration of the visual pigment back to the ground state requires recycling of its chromophore from the "bleached" all-trans retinal to the light-sensitive 11-cis retinal. This process, known as the visual cycle, requires export of the alltrans chromophore out of the photoreceptors and its conversion to the 11-cis form in retinal pigment epithelial (RPE) cells (for both rods and cones) or in retinal Müller glia (for cones only). The 11-cis chromophore is then imported back into photoreceptors, where it combines with a molecule of free opsin to regenerate the visual pigment $(1,2)$. The cone-specific visual cycle (3) has been suggested to enable cones, but not rods, to quickly recover from bright light exposure and to function over a wide range of light intensities (4-6). While an active area of research $(7,8)$, to date, none of the putative molecular components in this pathway have been shown to actually affect mammalian cone function, casting doubt on the significance of this pathway.

Cellular retinaldehyde-binding protein (CRALBP) is a retinoid-binding protein expressed in RPE and Müller glia and believed to be involved in the retinal visual cycle (1). CRALBP is a $36-\mathrm{kDa}$ water-soluble protein with 2 conformational states facilitating the intracellular transport of hydrophobic 11-cis retinoids (9). In zebrafish, 2 distinct orthologs, cralbp $a$ and cralbp $b$, are expressed in RPE and Müller cells, respectively $(10,11)$. Notably, knockdown

Conflict of interest: The authors have declared that no conflict of interest exists. Submitted: October 23, 2014; Accepted: December 11, 2014.

Reference information: / Clin Invest. 2015;125(2):727-738. doi:10.1172/JCI79651. of either of the 2 isoforms leads to decreased cone-driven electroretinographic (ERG) responses (10), suggesting a role of Müller cell-expressed CRALBP in zebrafish cone function (see also ref. 12). In mammals, CRALBP is encoded by a single gene, Rlbp1, expressed in both RPE and Müller cells. Mutations in human RLBP1 cause several autosomal recessive retinal diseases, such as autosomal recessive retinitis pigmentosa (13), Bothnia dystrophy (14-16), retinitis punctata albescens (17), fundus albipunctatus $(18,19)$, and Newfoundland rod-cone dystrophy $(20)$. These visual disorders are characterized by early-onset night blindness and may be followed by functional defects in the macular region (21). CRALBP is required for the proper function of the RPE visual cycle and for the timely recovery of mammalian rod and cone ERG responses (22). However, the role of Müller cell-expressed CRALBP in the mammalian retinal visual cycle is unknown. It is also not clear whether CRALBP in RPE, Müller cells, or both is required for the normal function of mammalian cones. Here, we used behavioral and electrophysiological assays in $\mathrm{Rlbp1}^{-/}$mice to examine the overall effect of CRALBP deletion on M-cone function. We also used molecular tools to explore the mechanism by which the lack of CRALBP causes cone function deterioration. We then used adeno-associated virus-mediated (AAV-mediated) gene transfer to selectively restore CRALBP in RPE or Müller cells and examine the distinct roles of the 2 visual cycles in supporting mammalian $\mathrm{M}$-cone function.

\section{Results}

The deletion of CRALBP suppresses mammalian cone visual function. In addition to causing well-documented rod-driven scotopic visual disorders $(15,17)$, mutations in CRALBP also disrupt conedriven photopic vision in humans $(13,16,20)$. It is believed that 
A

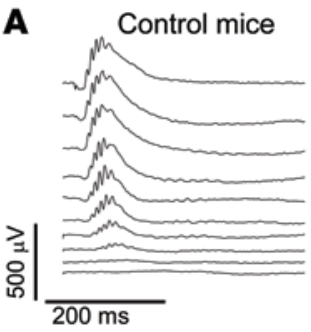

B

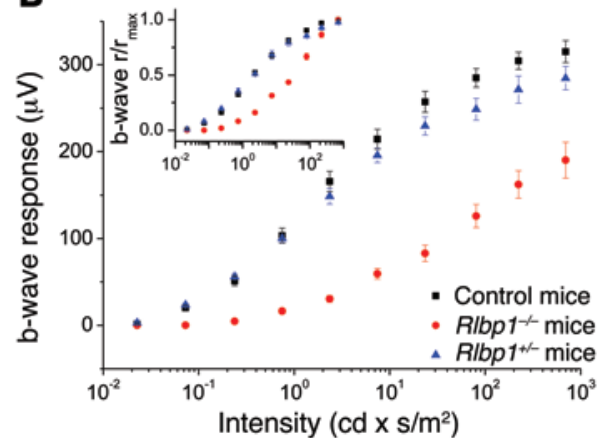

$R / b p 1^{-\%}$ mice

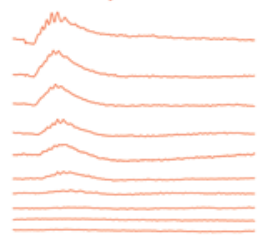

C

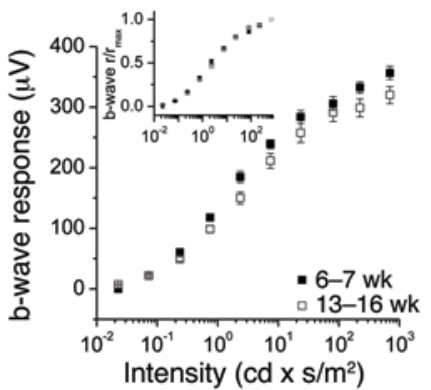

D

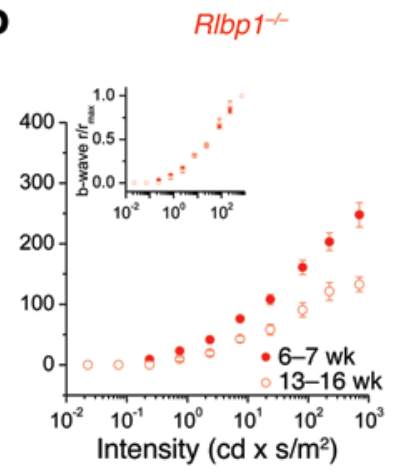

Figure 1. Deletion of CRALBP reduces photopic in vivo ERG-response amplitude and sensitivity. (A) Representative in vivo cone ERG responses from control (black traces), $R / b p 1^{-/-}$(red traces), and $\mathrm{Rlbp1}^{+/-}$(blue traces) mice. Test flash intensities increased from $2.27 \times 10^{-2} \mathrm{~cd} \times \mathrm{s} / \mathrm{m}^{2}$ (bottom traces) to 697 $\mathrm{cd} \times \mathrm{s} / \mathrm{m}^{2}$ (top traces) in steps of approximately $0.5 \mathrm{log}$ units. (B) Ensemble-averaged cone b-wave intensity-response curves for control $(n=10), R / b p 1^{1 /-}$ $(n=12)$, and $R / b p 1^{+/-}(n=10)$ mice. (C) Cone b-wave intensity-response curves for control mice aged 6-7 weeks (black squares, $\left.n=8\right)$ and 13-16 weeks (white squares, $n=10$ ). (D) Cone b-wave intensity-response curves for Rlbp1 $1^{-1-}$ mice aged 6-7 weeks (red circles, $n=6$ ) and 13-16 weeks (white circles, $n=6$ ). Insets in B-D show the corresponding normalized $\left(r / r_{\max }\right)$ intensity-response curves. Results represent the mean $\pm S E M$.

the pathophysiology for both rods and cones is based on inefficient chromophore recycling $(13,14)$. However, the exact mechanisms of cone dysfunction in CRALBP-related diseases and the relative contributions of the RPE visual cycle and the retinal visual cycle to cone dark adaptation are unknown. To address these questions, we first examined how the deletion of CRALBP affects the conedriven photopic visual performance of CRALBP-knockout mice by optomotor response behavioral tests. All functional experiments were performed with knockout mice lacking the rod transducin $\alpha$ subunit $\left(\right.$ Gnat1 $\left.^{-/}\right)$. This facilitated the isolation of cone function by ablating rod photoresponses while preserving normal retina morphology and cone function $(6,23)$. The experiments were performed with LCD monitor white light, which would be expected to selectively activate mouse M-cones (peak absorption at $508 \mathrm{~nm}$ ) but not S-cones (peak absorption at $360 \mathrm{~nm}$ ) (24). We found that the background light intensity required to achieve half-maximal cone-driven contrast sensitivity in 6-week-old $\mathrm{Rlbp1}^{-/-}$mice was approximately 10 -fold higher than that required for controls $(-0.4$ and $-1.3 \log \mathrm{cd} \times \mathrm{s} / \mathrm{m}^{2}$, respectively). Thus, the absence of CRALBP caused a substantial desensitization of cone-driven vision.

We then asked whether the vision loss observed at a behavioral level was caused by the deterioration of cone function in the absence of CRALBP. We conducted in vivo ERG recordings to examine the dark-adapted cone b-wave responses of 6- to 13-weekold $R l b p 1^{-/-}$mice. We used 530-nm LED flashes to selectively excite M-cones (24) up to the system's $25 \mathrm{~cd} \times \mathrm{s} / \mathrm{m}^{2}$ intensity limit and Xenon flash for higher intensities. We observed a significant $40 \%$ $\left(P<10^{-4}\right)$ decrease in the maximal M-cone b-wave amplitude in $R l b p 1^{-/-}$mice compared with that in both $\mathrm{Rlbp1}^{\mathrm{I}^{++}}$(control) and $R l b p 1^{+/-}$mice (Figure 1, A and B). In addition, we found a dramatic 20-fold decrease in photopic sensitivity, as measured by the corresponding increase in $\mathrm{I}_{1 / 2}$ (the flash intensity required to achieve a half-maximal response) of the $R l b p 1^{-/-}$cone b-wave responses (Figure 1B). Surprisingly, light sensitivity in the absence of CRALBP was diminished to such an extent that even the brightest light stimulus of the ERG system ( $697 \mathrm{~cd} \times \mathrm{s} / \mathrm{m}^{2}$, Xenon flash) could not generate a saturated photopic b-wave response (Figure 1B, red circles). In contrast, light sensitivity in $R l b p 1^{+/-}$mice was comparable to that in control mice (Figure 1B, blue triangles, inset).

In the course of these recordings, we noticed that the older $R l b p 1^{-1-}$ mice had smaller cone b-wave amplitudes than did the young adult mice. We compared the ERG b-wave responses from 6- to 7-week-old and 13- to 16-week-old mice to examine the long-term effect of CRALBP knockout on mouse photopic vision. In control animals, cone b-wave amplitude showed an approximately $10 \%$ (NS) reduction with age (Figure $1 \mathrm{C}$ ). In contrast, the cone b-wave amplitude of $R l b p 1^{-/-}$mice decreased by nearly $50 \%$ $(P<0.001)$ over the same 2-month period (Figure 1D). The light sensitivity, as estimated from the $\mathrm{I}_{1 / 2}$ of the respective normalized intensity-response curves, remained unchanged for both groups within the 2 months of aging (Figure $1, C$ and D, insets). Thus, the deletion of CRALBP caused a dramatic and progressive reduction in cone-driven visual performance.

The deletion of CRALBP desensitizes mammalian cones and lowers their phototransduction amplification. The reduced photopic b-wave amplitude and sensitivity of CRALBP-deficient mice could be caused by a deficit either in cone phototransduction or in cone-tobipolar cell synaptic transmission. To distinguish between the 2 possibilities, we determined whether cone phototransduction was affected directly by the deletion of CRALBP by performing ex vivo 

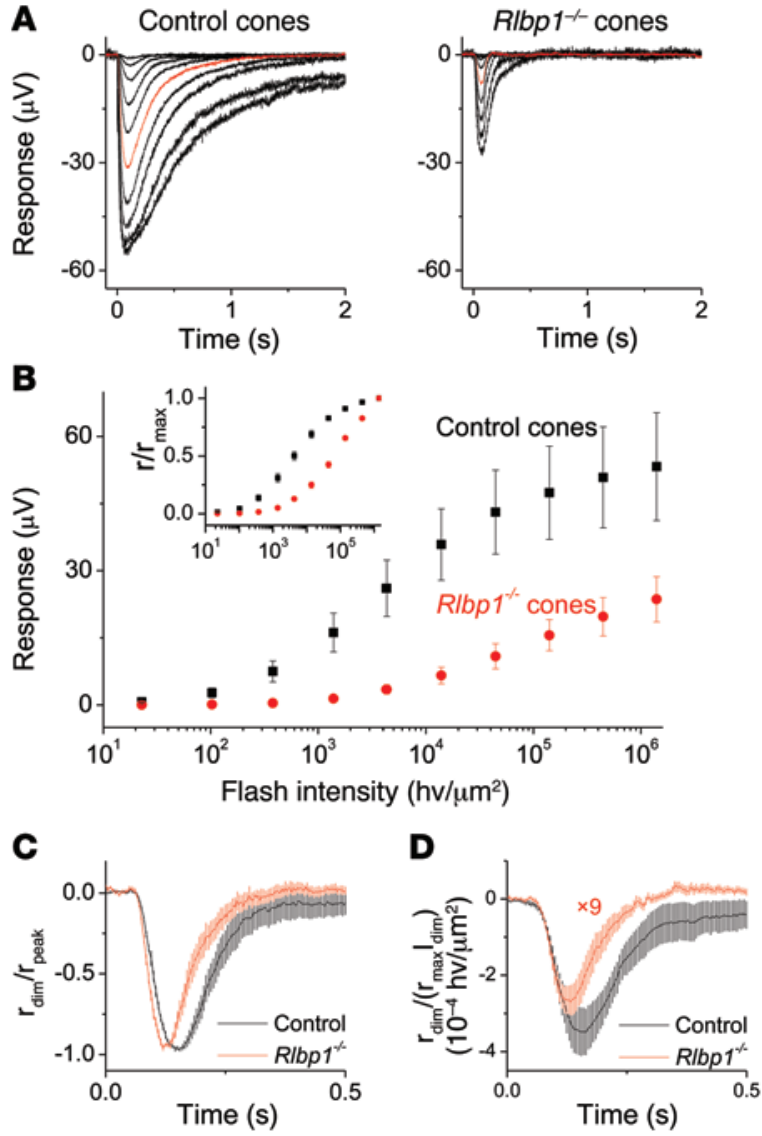

recordings from isolated retina. This technique allowed us to pharmacologically block synaptic transmission (see Methods) and isolate the cone (a-wave) response. We used a 505-nm LED flash light to stimulate the M-cones in these recordings. Similar to the in vivo ERG b-wave results above, the ex vivo transretinal responses from $\mathrm{Rlbp1}^{-/}$cones were smaller than those from control cones, with a greater than $50 \%(P<0.05)$ decrease in maximal amplitude (Figure 2, A and B). Indicative of their reduced sensitivity, the responses of $\mathrm{Rlbp1}^{-1-} \mathrm{M}$-cones could not be saturated, even at the maximal possible light intensity of our system (Figure 2B). The analysis of their corresponding intensity-response functions showed a 20-fold lower sensitivity (higher $\mathrm{I}_{1 / 2}$ ) compared with that in control cones (Figure 2B, inset). The absence of CRALBP also resulted in a somewhat accelerated cone response inactivation (Figure $2 \mathrm{C}$ ). In addition, consistent with their reduced light sensitivity, CRALBP-deficient cones had a 9-fold smaller phototransduction amplification compared with that in control cones (Figure 2D), revealed by the corresponding scaling factor required to match the rising slopes of the fractional dim flash responses to 103 photons $/ \mu \mathrm{m}^{2}$ for control cones and 1,387 photons $/ \mu \mathrm{m}^{2}$ for $R l b p 1^{-/-}$cones. Taken together, these results demonstrate that deletion of CRALBP in mice leads to severe desensitization and altered cone phototransduction in dark-adapted M-cones, which in turn produces a desensitized cone b-wave and suppressed cone-mediated vision.

Deletion of CRALBP severely impairs mammalian cone dark adaptation. We next sought to determine the effect of CRALBP deletion on RPE and retina visual cycles in the context of cone dark adaptation. First, we determined the overall effect of CRALBP deletion on
Figure 2. Deletion of CRALBP reduces transretinal cone-response amplitude and sensitivity. (A) Representative transretinal cone responses from control (left panel) and $\mathrm{Klbp}^{-1-}$ (right panel) retinae. Test flash intensities increased from 23 photons $/ \mu \mathrm{m}^{2}$ to $1.40 \times 10^{6}$ photons $/ \mu \mathrm{m}^{2}$ in steps of $0.5 \log$ units. For both panels, the flash intensity producing the response shown in red was $1.39 \times 10^{4}$ photons $/ \mu m^{2}$. (B) Ensemble-averaged absolute and normalized (inset) cone intensity-response curves for control $(n=13)$ and $R^{\prime b p T^{--}}(n=13)$ retinae. (C) Ensemble-averaged normalized cone dim flash responses from control $(n=12)$ and $R / b p 1^{-1-}(n=13)$ retinae. (D) Ensemble-averaged dim flash responses, r, from control $(n=13)$ and Rlbp1 $^{-1-}(n=11)$ cones normalized to the maximal response, $r_{\text {max }}$, and to flash intensity and with matched rising slopes to determine the change in phototransduction amplification. Results are shown as the mean \pm SEM.

cone dark adaptation. Using in vivo ERG recordings, we examined the cone b-wave sensitivity recovery of control and $\mathrm{Rlbp1}^{-/-}$mice following exposure to a brief bright-green light estimated to photoactivate (bleach) $90 \%$ of the M-cone visual pigment (see Methods for details). As expected, cone b-wave sensitivity in the control mice underwent robust recovery following the bleaching and returned within 50 minutes to an estimated $50 \%$ of the pre-bleaching level (Figure 3A, black squares). An incomplete photoreceptor dark adaptation after bleaching, as detected in ERG recordings of WT mice, is not unusual (6) and is most likely caused by the general anesthetics (25). In striking contrast to the ERG response recovery in control mice, $\mathrm{M}$-cones in $\mathrm{Rlbp}^{-/-}$mice recovered only a slight fraction of their sensitivity following an identical bleaching (Figure $3 \mathrm{~A}$, red circles). Thus, cone dark adaptation in vivo, driven through the combined action of the RPE and Müller cell visual cycles, was severely compromised by the deletion of CRALBP. Notably, the effect of CRALBP deletion on the recovery of cone sensitivity was more pronounced than the previously reported delay in recovery of cone b-wave-response amplitudes (22).

Next, to determine the specific effect of CRALBP deletion on the Müller cell visual cycle, we performed cone dark-adaptation experiments in retina dissected free of RPE, in which cone pigment regeneration can be driven only by the retinal visual cycle. After an initial greater than 100-fold desensitization caused by the bleaching, within seconds, cones in both control and $R l b p 1^{-/-}$ retinae showed a rapid initial increase in sensitivity (Figure 3B). This partial recovery, which was most likely due to inactivation of the phototransduction cascade following the bleaching, was comparable in the 2 mouse strains. However, the $\mathrm{Rlbp1}^{-/-}$cone sensitivity failed to recover further during the 12 minutes of postbleach recordings, while control cones recovered to within 5 -fold of their pre-bleaching level (Figure 3B). We conclude that deletion of CRALBP has a dramatic effect on the ability of the retinal visual cycle to promote mouse cone dark adaptation. Taken together, these results demonstrate that CRALBP plays a role in both the RPE and retinal visual cycles and that its deletion severely impairs the ability of both pathways to promote mammalian cone dark adaptation.

Dark rearing of CRALBP-deficient mice restores cone function but not cone dark adaptation. It is believed that the role of CRALBP in both the RPE and retinal visual cycles is to accelerate the production of 11-cis retinoid (1). We hypothesized that in the absence of CRALBP, both visual cycles would still remain functional but would fail to provide sufficient chromophore for sustaining normal 


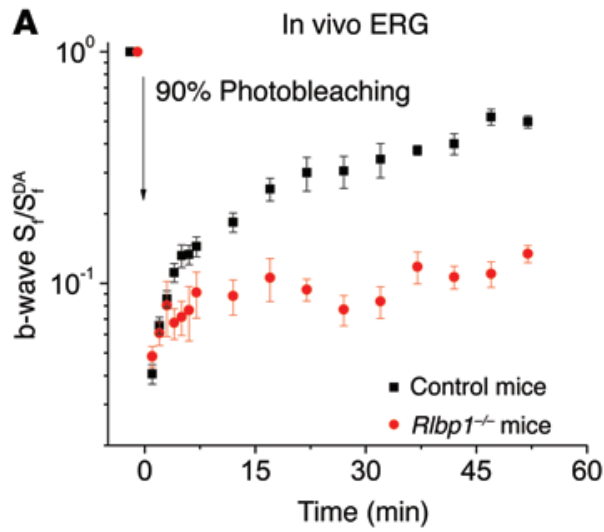

Figure 3. The deletion of CRALBP suppresses cone dark adaptation. (A) Normalized cone b-wave sensitivity (b-wave $S_{f} /$ b-wave $S_{f}^{D A}$ ) from in vivo ERC recordings during dark adaptation following $90 \%$ pigment bleaching at $t=0$ for control $(n=10)$ and $R / b p 1^{-/-}(n=10)$ mice. (B) Cone sensitivity, $\mathrm{S}_{\mathrm{f}}$, normalized to its dark-adapted value, $\mathrm{S}_{f}^{\mathrm{DA}}$, from transretinal recordings during dark adaptation following $90 \%$ pigment bleaching at $t=0$ for control $(n=9)$ and $R / b p 1^{-1-}$ $(n=10)$ isolated retinae. Results represent the mean \pm SEM cone function in 12-hour light/12-hour dark cyclic conditions. To test this idea, we first attempted to restore cone function in $\mathrm{Rlbp1}^{-1-}$ mice by supplying them with exogenous chromophore in order to regenerate any free cone opsin into visual pigment. However, the application of either 9-cis retinal in vivo (Figure 4A) or 11-cis retinal ex vivo (Figure 4B) failed to rescue M-cone sensitivity. In contrast, treatment of chromophore-deficient $R p e 65^{-/-}$retinae with exogenous 11-cis retinal ex vivo and application of 9-cis retinal to $R p e 65^{-/-}$mice in vivo resulted in a robust increase in rod sensitivity and maximal response (data not shown), as previously reported $(26,27)$. These results demonstrate that free opsin is not present in $R l b p 1^{-/-}$cones in detectable amounts and therefore is not the cause for the reduced sensitivity and response amplitude of cones in CRALBP-deficient mice.

We next examined whether the suppressed M-cone function in CRALBP-knockout mice is caused by a long-term chromophore deficiency. We raised $R l b p 1^{-/-}$newborn mice in near-complete darkness to substantially slow down the consumption of chromophore in their eyes and lower the demand for recycled chromophore by the cones. It was recently shown that raising mice in complete darkness leads to an eventual decline in cone-tobipolar cell synaptic transmission (28). However, the occasional
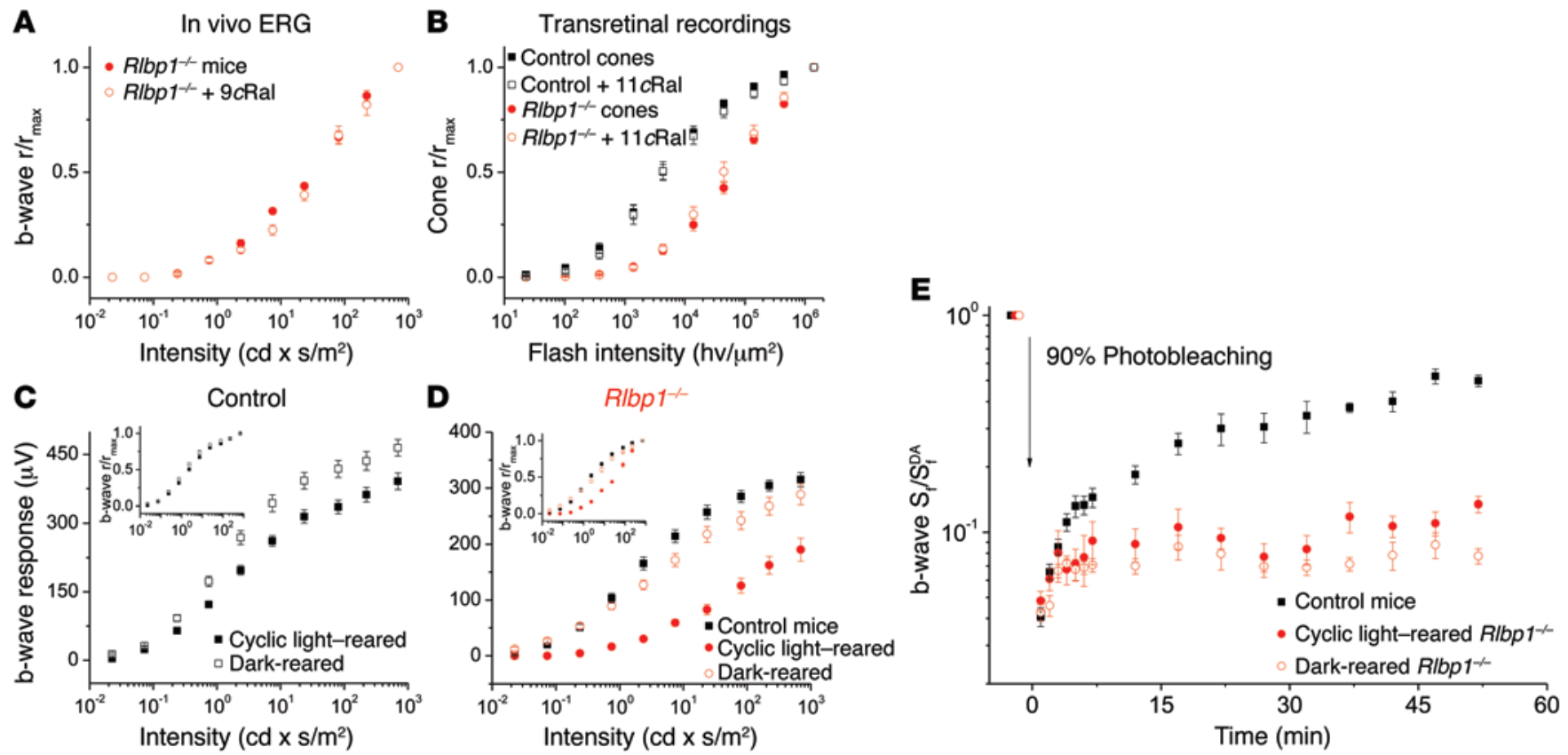

Figure 4. Dark rearing, but not acute treatment with exogenous chromophore, rescues CRALBP-deficient cone sensitivity. (A) Normalized in vivo ERC cone b-wave intensity-response curves for untreated control (replotted from Figure 2B inset) and 9-cis retinal-treated $(n=6) R / b p 1^{-1-}$ mice. (B) Normalized transretinal cone intensity-response curves for control (black, $n=6)$ and $R / b p 1^{-1-}($ red, $n=6)$ retinae in control solution (filled symbols; replotted from Figure 3B inset) and after treatment with exogenous 11-cis retinal (open symbols, $n=6$ ). 9cRal, 9-cis retinal; 11cRal, 11-cis retinal. (C) Cone b-wave intensity-response curves from in vivo ERG recordings of control mice raised in cyclic light (black squares, $n=14$ ) or in darkness (white squares, $n=10$ ). (D) Cone b-wave intensity-response curves from in vivo ERG recordings of control (black squares; replotted from Figure $1 \mathrm{~B}$ ) and $R / b p 1^{-/-}$mice raised in cyclic light (red filled circles; replotted from Figure $1 \mathrm{~B}$ ) and $R / \mathrm{lbp}^{1-1-}$ mice raised in darkness (open red circles, $n=10$ ). Insets in $\mathbf{C}$ and $\mathbf{D}$ show the corresponding normalized intensity-response curves. (E) Normalized cone b-wave sensitivity (b-wave $S_{f} / b$-wave $S_{f}{ }^{D A}$ ) from in vivo ERG recordings during dark adaptation following $90 \%$ pigment bleaching at $t=0$ for control (black squares) and $R / b p 1^{-1-}$ mice raised in cyclic light (filled red circles; replotted from Figure 4A) and for $R / b p^{-1-}$ mice raised in darkness (open red circles, $n=10$ ). Results represent the mean \pm SEM. 
A
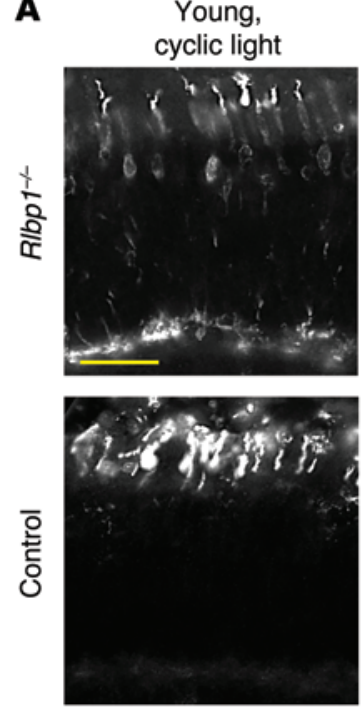

B
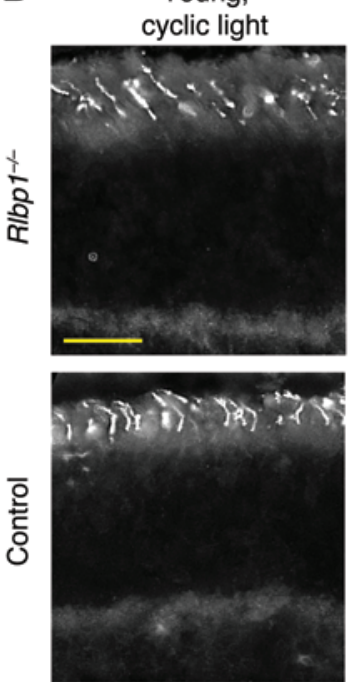

Old, cyclic light
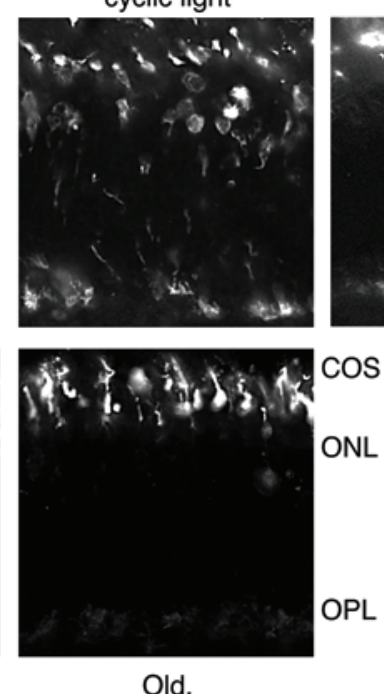

Old, cyclic light
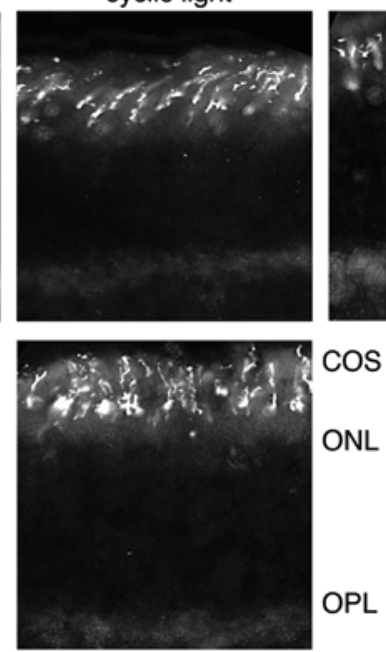

Young,

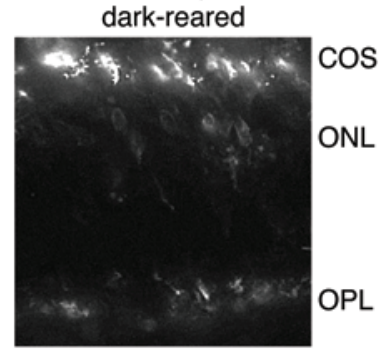

$\cos$

ONL

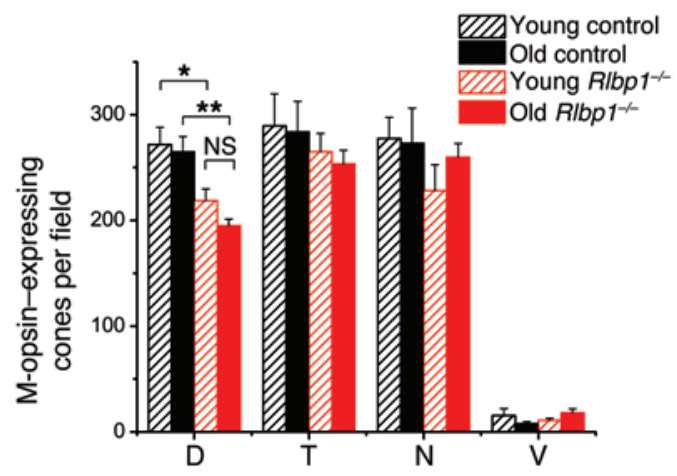

D

Young,

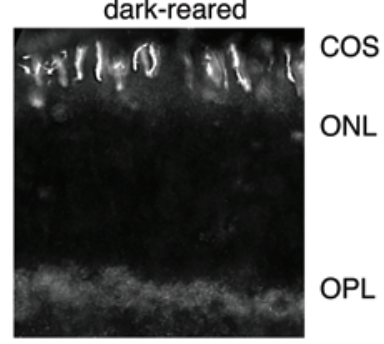

ONL

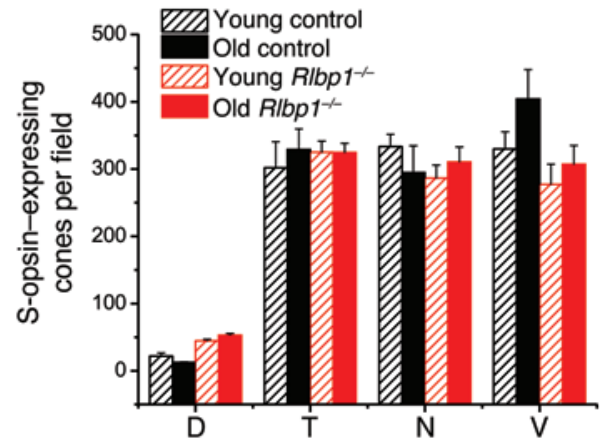

Figure 5. Deletion of CRALBP affects the localization of $\mathbf{M - o p s i n ~ a n d ~ n u m b e r ~ o f ~ c o n e s ~ e x p r e s s i n g ~} \mathbf{M}$-opsin. Antibody staining of retinal frozen sections from Rlbp1/- and control mice for (A) M-opsin and (B) S-opsin. Representative images are shown. At least 3 retinae per condition were examined. Scale bars: $25 \mu \mathrm{m}$. COS, cone outer segment; ONL, outer nuclear layer; OPL, outer plexiform layer. For clarity, the DAPI channel is not shown. (C) Quantification of whole-mount M-opsin antibody staining ( $n=4$ retinae per condition). ${ }^{*} P<0.05,{ }^{*} P<0.01$ by unpaired 2 -tailed Student's $t$ test. (D) Quantification of whole-mount S-opsin antibody staining ( $n=3$ retinae per condition). D, dorsal; T, temporal; N, nasal; V, ventral. Young, 6- to 7-week-old mice; Old, 3- to 6-month-old mice. Results represent the mean \pm SEM.

brief exposure to room light during routine animal care was sufficient to maintain normal cone function in our control mice and resulted in only a slight increase in the maximal amplitude of their in vivo ERG b-wave response (Figure 4C). Importantly, the photopic b-wave sensitivity of control mice, as measured from their normalized intensity-response curve, was unchanged by the dark rearing (Figure $4 \mathrm{C}$, inset). In contrast, raising $R l b p 1^{-/-}$mice in darkness not only restored cone b-wave maximal response (Figure 4D), but also boosted cone sensitivity levels to those of control cones (Figure 4D, inset). However, a subsequent exposure to bleaching light unmasked the deficiency in cone pigment regeneration, as the dark adaptation in CRALBP-deficient mice was identical for animals raised in darkness and in cyclic light (Figure 4E). Together, these results demonstrate that the dark rearing of $\mathrm{Rlbp}^{-/-}$mice slows down the consumption of chromo- phore enough to preserve cone function. However, dark rearing alone does not address the underlying deficit in the RPE and/or retinal visual cycles.

Deletion of CRALBP induces $M$-opsin mislocalization and loss of M-cones. What is the molecular mechanism underlying the functional deterioration in CRALBP-deficient cones? Based on the physiological results above, we hypothesized that CRALBP was required for proper localization of opsin protein. To test this hypothesis, we stained frozen sections from $\mathrm{Rlbp}^{-/-}$and control retinae with cone opsin antibodies. Whereas M-opsin was localized to the cone outer segment of the control retinae as expected, we observed striking mislocalization of $\mathrm{M}$-opsin to the cone cell bodies, axons, and pedicles of young (6-week-old) and old (4to 6-month-old) $R l b p 1^{-/-}$mice raised conventionally in cyclic light-dark conditions. Intriguingly, dark rearing of $\mathrm{Rlbp}^{-/-}$mice 

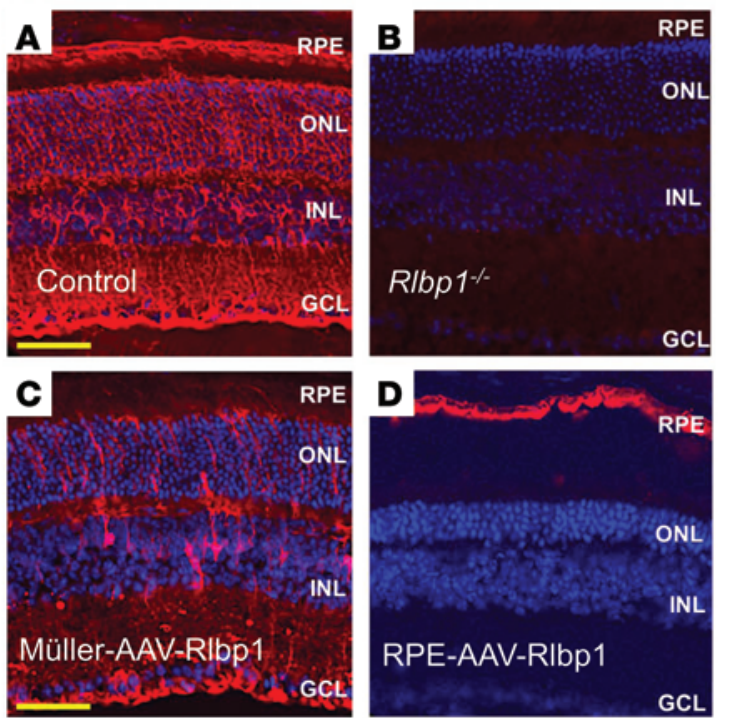

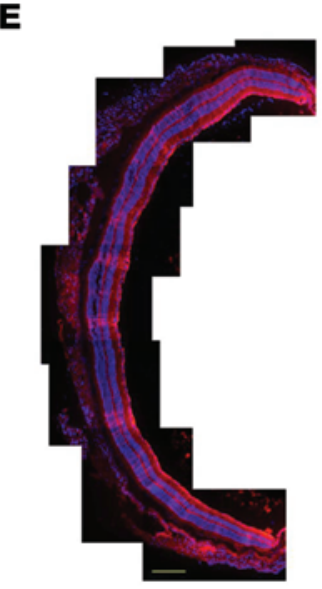

Müller-AAV-RLBP1

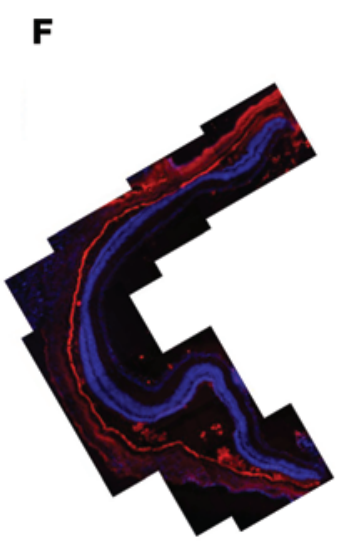

RPE-AAV-RLBP1

Figure 6. AAV-mediated delivery of CRALBP to Müller cells or RPE cells. Antibody staining shows expression pattern of CRALBP in (A) control retina, (B)

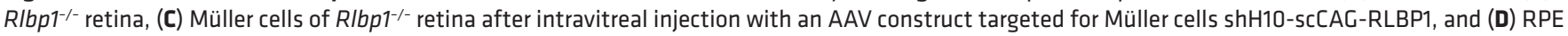
of $\mathrm{RIbp1}^{-1-}$ retina after intravitreal injection with an AAV construct targeted for RPE 7m8-scVMD2-RLBP1. Scale bars: $50 \mu \mathrm{m}$. INL, inner nuclear layer; GCL, ganglion cell layer. Widespread infection across the retina was achieved for both constructs, as seen in tiled images for (E) shH10-scCAG-RLBP1 and (F) 7m8-scVMD2-RLBP1. Red channel, anti-CRALBP; blue channel, DAPI. Scale bar: $200 \mu \mathrm{m}$.

appeared to ameliorate $\mathrm{M}$-opsin mislocalization to some extent (Figure 5A), consistent with the physiology results above. In contrast to $\mathrm{M}$-opsin, $\mathrm{S}$-opsin was appropriately localized to the cone outer segments in the retinae of both $R l b p 1^{-/-}$and control mice (Figure $5 \mathrm{~B}$ ). We also wondered whether the M-opsin mislocalization in $\mathrm{Rlbp1}^{-/-}$retinae was correlated with M-cone numbers. Quantification of whole-mount antibody staining revealed a lower density of M-opsin-expressing cones in the dorsal retinae of $\mathrm{Rlbp1}^{-1-}$ mice compared with dorsal retinae in age-matched controls (Figure $5 \mathrm{C}$ ), whereas the density of S-opsin-expressing cones was not affected (Figure 5D). Thus, the deletion of CRALBP resulted in both mislocalization of M-opsin and M-cone loss. However, overall, the cone density in $R l b p 1^{-/-}$retinae did not change markedly with age, suggesting that the observed age-dependent decline in cone function was caused by progressive deterioration in the light responses of individual $\mathrm{M}$-cones.

The retinal visual cycle is essential for cone function. In the above results, we demonstrated that CRALBP was crucial for maintaining normal $\mathrm{M}$-cone function in a cyclic light environment and for proper cone dark adaptation. However, because in these experiments CRALBP was absent in both RPE and Müller cells, we were not able to determine the relative contribution of each visual cycle to maintaining normal cone function. To address this question, we used 2 separate AAV vectors to express CRALBP specifically in either RPE or Müller cells of adult $R l b p 1^{1^{-/}}$mice (see Methods for details). First, we verified that CRALBP was delivered via intravitreal AAV injection to the RPE (via the RPE-specific AAV construct 7m8-scVMD2-RLBP1) or to the Müller cells (via the Müller cell-specific AAV construct shH10-scCAG-RLBP1) in $\mathrm{Rlbp1}^{-/-}$retinae. As previously shown (22), antibody staining revealed robust CRALBP expression in the RPE and Müller cells of WT mice (Figure 6A) and its complete absence in $\mathrm{Rlbp}^{-/-}$eyes (Figure 6B). Notably, immunohistochemistry revealed expression of CRALBP specifically in the targeted cell type for both AAV constructs (Figure 6, C and D). Moreover, the extent of intravitreal AAV infection appeared to be widespread, as demonstrated by the CRALBP expression in the targeted cell type along the length of the retina (Figure 6, E and F).

We then examined how the rescue of each visual cycle affected the dark-adapted function of M-cones as well as their ability to recover light sensitivity rapidly following a bleaching. The transretinal recordings revealed that dark-adapted cone sensitivity in Müller cell CRALBP-expressing $R l b p 1^{-/}$mice was improved by approximately 10-fold (Figure 7A) and that amplification of cone phototransduction was enhanced by 5.8 -fold compared with controls (Figure 7B). In contrast, dark-adapted cone responses from $\mathrm{RPE}$ cell CRALBP-expressing $R l b p 1^{-/-}$mice were indistinguishable from those of their AAV-GFP-injected littermates (Figure 7, A and B). This result demonstrates that expression of CRALBP in Müller cells is required for the normal function of dark-adapted $\mathrm{M}$-cones. The dim flash-response kinetics, cone maximal response, and cone b-wave maximal response were not affected by either RPE or Müller cell expression of CRALBP (data not shown), indicating an incomplete rescue of cone function. We also note that AAVmediated CRALBP delivery to either RPE or Müller cells failed to correct the M-opsin mislocalization defect (data not shown). Notably, in vivo cone dark adaptation was markedly improved by the AAV-mediated CRALBP rescue of either visual cycle with indistinguishable time courses (Figure 7C). In contrast, only the expression of CRALBP in Müller cells resulted in the rescue of cone dark adaptation in the isolated RPE-free retina, whereas expression of CRALBP in RPE had no effect on cone dark adaptation under these conditions (Figure 7D). Together, these results demonstrate the role of the retinal visual cycle in supporting mammalian M-cone function and indicate that CRALBP in Müller cells plays a key role in this pathway. 

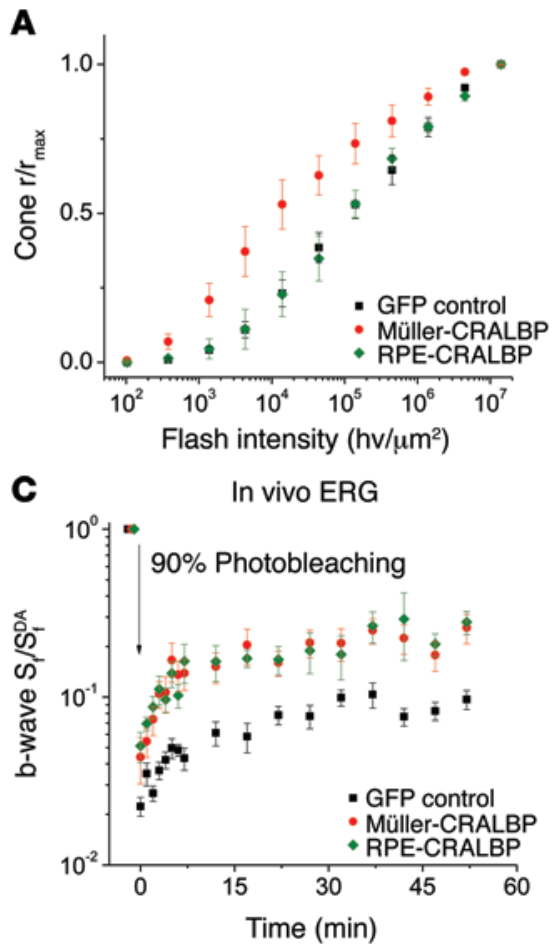
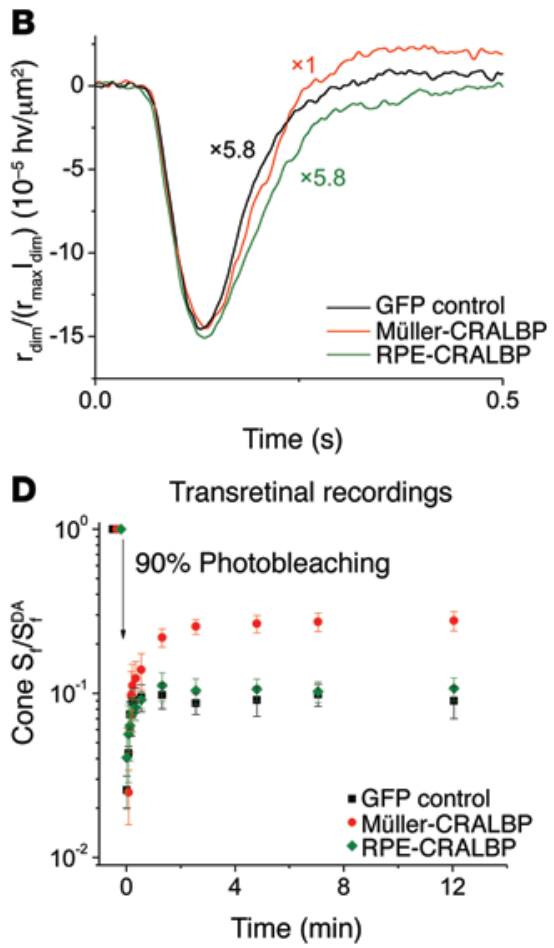

Figure 7. AAV-driven expression of CRALBP in Müller cells rescues the sensitivity and dark adaptation of CRALBP-deficient cones. (A) Ensemble-averaged transretinal cone intensity-response curves for RIbp1 $1^{-/-}$mice injected with AAV driving expression of GFP in RPE or Müller cells (black squares, $n=4$ ), CRALBP in Müller cells (red circles, $n=5$ ), and CRALBP in RPE cells (green diamonds, $n=3$ ). (B) Transretinal dim flash responses of $R / b p 1^{-/-}$mice showing the relative amplification for AAV-driven control GFP (black, $n=4$ ), Müller cell-specific CRALBP (red, $n=5$ ), and RPE-specific CRALBP (green, $n=3$ ) expression. (C) In vivo ERG recordings of cone b-wave dark adaptation (b-wave $S_{f} /$ b-wave $\mathrm{S}_{f}^{\text {DA) }}$ after a $90 \%$ bleaching in $R / b p 1^{1--}$ mice with AAVdriven expression of control GFP (black, $n=11$ ), Müller cell-specific CRALBP (red, $n=12$ ), and RPE-specific CRALBP (green, $n=8$ ). (D) Transretinal recordings of cone dark adaptation $\left(\mathrm{S}_{f} / \mathrm{S}_{f}{ }^{D A}\right)$ after a $90 \%$ bleaching of $R / \mathrm{lbp}^{-1-}$ retinae with AAV-driven expression of control GFP (black, $n=4$ ), Müller cell-specific CRALBP (red, $n=5$ ), and RPE-specific CRALBP (green, $n=3$ ). Results represent the mean \pm SEM.
Deletion of CRALBP affects the pupillary light reflex. Besides rods and cones, another cell type in the retina that requires chromophore for its function is the intrinsically photosensitive retinal ganglion cell (ipRGC) (29). CRALBP in Müller cells was recently proposed to facilitate the supply of 11-cis retinal to ipRGCs (30). We investigated this possibility by evaluating the effect of CRALBP deletion on light-driven pupil constriction. Rods in both control and $\mathrm{Rlbp1}^{-/}$mice do not respond to light due to the absence of GNAT1, and their pupillary light reflex is therefore mediated by the 2 remaining light-sensitive cell types in the retina: cones and ipRGCs (31). In melanopsin-knockout animals that lack melanopsin phototransduction but maintain normal cone function, there is a clear reduction in the magnitude of the pupillary light reflex, but only at high light intensities (32). To determine whether melanopsin phototransduction is affected in $\mathrm{Rlbp1}^{-/}$mice, we exposed 1 eye to light and measured pupil constriction in the contralateral eye (Figure 8A). This enabled us to evaluate the overall sensitivity of the pupillary light reflex. Two-way ANOVA analysis showed an overall significant difference $(P<0.001)$ between the pupil constriction intensity-response curves of control and $\mathrm{Rlbp1}^{-/-}$mice. Further 1-way ANOVA statistical analysis followed by Bonferroni's correction on the $P$ values at each intensity revealed a significantly higher $(P<0.05)$ threshold for pupil constriction in llbp $^{-1 /}$ mice compared with that in controls (Figure 8B). The light intensity required to reach $50 \%$ effective constriction $\left(\mathrm{EC}_{50}\right)$ was also slightly higher in CRALBP-deficient mice compared with that in controls, but the difference was not statistically significant (Figure $8 \mathrm{C}$ ). The maximal pupil constriction in bright light of $\mathrm{Rlbp1}^{-/-}$mice was comparable to that of controls, suggesting that the function of ipRGCs is largely unaffected by the deletion of CRALBP (32). In contrast, the maximal pupillary light reflex of Rpe65 ${ }^{--}$mice, in which all photoreceptor function is severely suppressed due to a lack of chromophore (33), was dramatically reduced (Figure 8, $\mathrm{A}$ and $\mathrm{B})$. This analysis suggests that the pupillary light reflex is overall different in $\mathrm{Rlbp1}^{-/-}$mice, with the difference restricted to relatively dim light levels, in which the pupil response is primarily driven by cone signals. The response at higher light intensities, which is dominated by the ipRGC signals, was not affected by the deletion of CRALBP. Together, these results indicate that CRALBP is not required for the delivery of chromophore to ipRGCs or for the regeneration of melanopsin and that the decreased sensitivity of the pupillary light reflex in $\mathrm{Rlbp1}^{-/-}$mice is most likely caused by suppressed cone function. However, a conclusive determination of this issue would require the generation of triple-knockout animals that lack both rod and cone phototransduction pathways in addition to a lack of the Rlbp1 gene.

\section{Discussion}

Molecular evidence for the function of a mammalian retinal visual cycle. The idea of a second, cone-specific visual cycle was first put forth in the 1970s (34) and has been gaining acceptance and experimental support in recent years $(3,4,35)$. This pathway was proposed to involve the conversion of spent all-trans retinol, released from cones, back into 11-cis retinol in the retinal Müller cells (3). Indeed, it was shown recently that the retina promotes pigment regeneration and dark adaptation in cones independently of the RPE, and that pharmacological ablation of Müller cells blocks this process (5). Recent biochemical studies have identified putative retinoid isomerase (7) and ester synthase activities (8) in Müller cells consistent with a retinal visual cycle. However, the molecular mechanism involved in the trafficking and recycling of chromophore still remains largely unknown, and skepticism still remains, partly due to the lack of experimental evidence for the involvement of any of the putative molecular players in this path- 

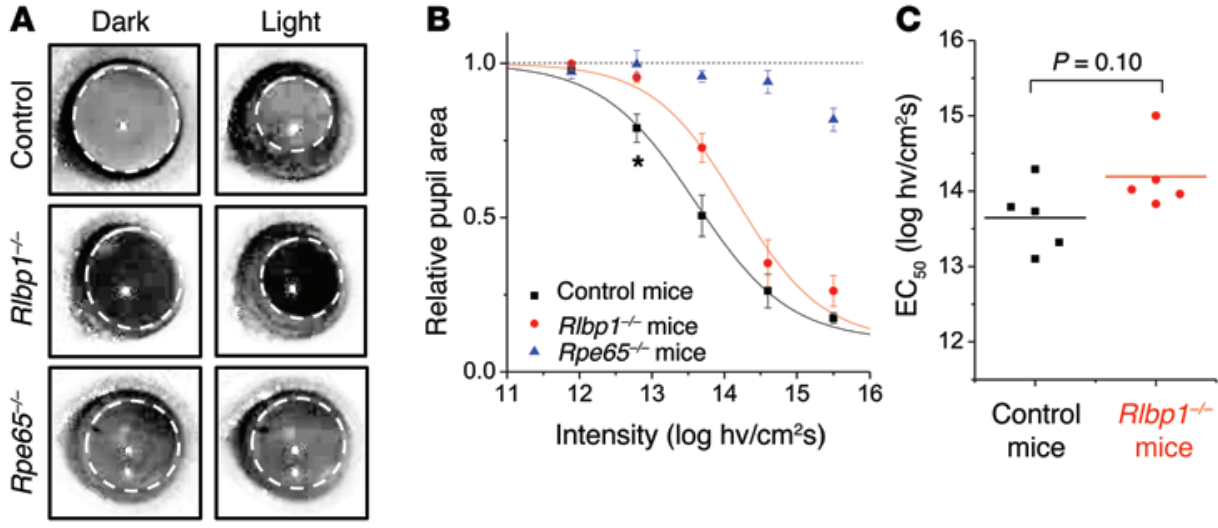

Figure 8. Deletion of CRALBP reduces the threshold of the pupillary light reflex. (A) Comparison of pupil size in darkness and in the light $\left(\sim 14 \log\right.$ photons $\left./ \mathrm{cm}^{2} / \mathrm{s}\right)$ in control, $R / b p 1^{-/-}$, and $R p e 65^{-/-}\left(\right.$Gnat $\left.^{1^{+/+}}\right)$negative control mice. (B) Averaged intensity-response curves for control $(n=5), \operatorname{Rlbp1}^{-1-}(n=5)$, and Rpe65 ${ }^{-/-}(n=4)$ mice. A significant difference was observed at threshold $\left({ }^{*} P<0.05\right)$ between $R / b p 1^{-1-}$ and control mice by 2-way ANOVA followed by Bonferroni's post test. Results represent the mean \pm SEM. (C) Intensity required to reach $\mathrm{EC}_{50}$ in control and $R / b p 1^{-1-}$ mice. $P=0.10$, NS, by 2-tailed unpaired Student's $t$ test.
(Figures 1 and 2). Notably, this suppressed cone function can be rescued by raising the animals in darkness (Figure 4). This result indicates that unlike the other widely studied chromophore-binding protein IRBP (39, 40), CRALBP is not required for the normal development and survival of cones. Instead, the preservation of normal cone function in dark-reared $R l b p 1^{-/-}$mice suggests that the cone phenotype is caused by inadequate chromophore supply. One possibility is that the delayed recycling of chromophore in the absence of CRALBP leads to chronic chromophore deficiency, so that even after overnight dark adaptation of these mice, the pigment content of their cones is still not fully restored. However, considway in actually controlling the function of cones. Here, we settle this question by demonstrating that the deletion of CRALBP in Müller cells prevents this pathway from promoting dark adaptation in mouse cones. Our results also reveal a previously unappreciated role of the retinal visual cycle in the long-term maintenance of normal mammalian cone function.

The role of CRALBP in the retinal visual cycle. We found that deletion of CRALBP in mice resulted in a dramatic suppression of cone dark adaptation both in vivo, when driven by the combined action of the RPE and retinal visual cycles (Figure 3A), and ex vivo, when driven only by the retinal visual cycle (Figure 3B). Therefore, suppression of the RPE visual cycle by deletion of CRALBP not only affects the rods, as previously shown by Saari et al. (22), but also compromises the ability of the RPE to drive the dark adaptation of cones. More important, our results also clearly demonstrate that CRALBP in Müller cells plays a similar role in the retinal visual cycle to promote cone dark adaptation.

What is the mechanism of CRALBP function in the retinal visual cycle? The rescue of cone function in dark-reared $R l b p 1^{-1-}$ mice indicates that deletion of CRALBP does not block the function of the retinal visual cycle; rather, CRALBP likely regulates its efficiency or kinetics. In RPE, CRALBP interacts with the isomerohydrolase to carry 11-cis retinol and facilitate its oxidation to 11-cis retinal (36). A recent study suggests that in vitro CRALBP closely interacts with DES1, the proposed retinoid isomerase in Müller cells (7). Therefore, it is possible that CRALBP takes up 11-cis retinol from DES1 and facilitates the reisomerization of chromophore by the retinal visual cycle. CRALBP also facilitates the release of chromophore from RPE cells and its subsequent uptake by photoreceptors $(37,38)$. Thus, a second possibility is that CRALBP plays a similar role in Müller cells and accelerates the flow of chromophore to cones.

The influence of chromophore deficiency on cone function. Rods in CRALBP-deficient mice have normal sensitivity and maximal response after overnight dark adaptation (22). In striking contrast, dark-adapted cones in CRALBP-deficient mice have significantly reduced response amplitude and 20-fold lower sensitivity ering that CRALBP-deficient rod responses are normal after 18 hours of dark adaptation (22), it is unlikely that only cones would be affected by such incomplete dark adaptation. Indeed, our observation that application of exogenous chromophore failed to rescue cone function (Figure 4) rules out this possibility.

An alternative hypothesis is that the chronic deficiency of chromophore affects cone opsin levels. Evidence for this notion comes from studies of a key enzyme in the RPE visual cycle, RPE65. In contrast to the slowed-down RPE visual cycle in $\mathrm{Rlbp1}^{-1-}$ mice, Rpe $65^{-/-}$mice lack this pathway completely and are unable to supply chromophore to their photoreceptors (41). This results in mislocalization of cone opsin and very rapid degeneration of the cones $(42,43)$, both of which are attributed to chromophore deficiency $(42,44)$. Consistent with the role of chromophore in supporting cone opsin folding and expression, a recent study demonstrated that proper cone opsin expression requires sufficient chromophore supply to the endoplasmic reticulum (45). In addition, 11-cis retinal in the inner segment of cones also appears to facilitate the transport of several phototransduction proteins to the cone outer segments (46). Thus, the deterioration of M-cone function that we observed in $\mathrm{Rlbp1}^{-/-}$mice is likely a direct result of the chromophore deficiency caused by impairment of the retinal visual cycle. Notably, we found that S-cone opsin localization and expression were not affected in $\mathrm{Rlbp1}^{-/-}$mice (Figure 5, B and D), indicating that apo S-opsin might be more stable than its $\mathrm{M}$-opsin counterpart. It is intriguing in this context that mouse $\mathrm{M}$-cones are more susceptible to age-dependent degeneration than are S-cones (47), suggesting that cone opsin stability might play a role in age-dependent cone degeneration. Regardless of the mechanism affecting the loss of cone function in $\mathrm{Rlbp1}^{-/-}$mice, the rescued cone function in animals raised in darkness suggests that minimizing light exposure might be a simple and effective approach for protecting cones from degeneration and preventing photopic vision loss in patients with CRALBP-based visual disorders.

The contribution of 2 visual cycles to cone function. By selectively rescuing either the RPE or Müller cell visual cycle using AAV-RLBP1 in $\mathrm{Rlbp1}^{-/-}$mice, we were able to identify the contribu- 
tion of each visual cycle to supporting normal cone sensitivity and dark adaptation. Our finding that only rescue of the retinal visual cycle, but not the RPE visual cycle, restores normal sensitivity of dark-adapted $R l b p 1^{-/-}$cones (Figure 7) reveals a previously unappreciated function of the retinal visual cycle and demonstrates that this pathway plays a crucial role in maintaining long-term cone function. One interesting unexplored possibility emerging from these results is that age-dependent decline in the efficiency of the Müller cell visual cycle contributes to the gradual loss of cone function and is linked to age-related cone visual disorders in patients. Thus, genetic or pharmacological treatments aimed at boosting the retinal visual cycle might have therapeutic benefit for age-dependent cone visual loss.

It has been suggested that cone dark adaptation is biphasic, with an initial fast recovery dominated by the retinal visual cycle and a slow subsequent recovery contributed by the RPE visual cycle (6). However, we found that the rescue of either of the 2 visual cycles in $\mathrm{Rlbp1}^{-/-}$mice results in cone dark adaptation in vivo with indistinguishable kinetics (Figure 7C). It is not clear at the moment whether this reflects a more complex interplay between the contributions of the 2 visual cycles than was previously appreciated, or a developmental compensatory modulation of 1 pathway in the absence of the other. Interestingly, neither of the rescues of the 2 visual cycles restored the maximal cone response, suggesting reduced cone number or phototransduction capacity. One possibility is that both visual cycles are required for normal cone function. Alternatively, it is possible that the loss of cone function in $\mathrm{Rlbp1}^{-/-}$mice is caused by chromophore deficiency at an early stage of development and therefore could not be rescued by AAV injections in adult animals. While the 2 hypotheses are not mutually exclusive, our aging experiments on $R l b p 1^{-/-}$mice (Figure 1) support the latter. Future studies with animals of different ages should resolve these questions and provide invaluable information about the therapeutic potential of such treatments. Notably, we were able to achieve selective and highly efficient CRALBP expression in Müller cells and even in the RPE (Figure 6F) with an intravitreal AAV injection.

\section{Methods}

Animals. CRALBP-deficient mice (22) were provided by John Saari (University of Washington, Seattle, Washington, USA). To facilitate cone recordings, CRALBP-knockout mice were crossed with rod transducin $\alpha$-knockout mice (Gnat1 ${ }^{-/}$) (23) obtained from Janis Lem (Tufts University, Boston, Massachusetts, USA) to eliminate rod responses. The role of CRALBP in cone function and morphology was then determined by comparing adult ( 6 weeks to 6 months of age) control $\left(\right.$ Gnat1 $\left.^{--}\right)$and Rlbp1 $^{-/}$(CRALBP-deficient Gnat1--) mice. All mice used in this study were confirmed to be free of the $r d 8$ mutation (48). For dark-rearing experiments, newborn mice were transferred to a light-proof cabinet and briefly exposed to ambient light only once a week during cage changing. All other mice were raised in a 60 Lux 12-hour light/12-hour dark cycle. Animals were dark adapted for 18 hours before electrophysiological recordings and at least 30 minutes prior to pupillary light reflex tests. The animals used for the optomotry tests were light adapted before the experiment. In addition, Rpe $65^{-/-}$ $\left(\right.$ Gnat $\left.^{+/+}\right)$mice were used as control animals for pupillary light reflex and exogenous chromophore treatment experiments.
Photopic vision measured from optomotor responses. The threshold of contrast sensitivity was measured with a commercially available OptoMotry System (CerebralMechanics) as previously described (6). The intensity of the background light of the system was controlled with a custom-made cylinder of neutral-density filter film, wrapped around the mouse stand. The contrast-sensitivity threshold at a 0.128 cycles/ degree grating spatial frequency was measured by an automated computer program when mice failed to provide optomotor responses. The tests started from brightest $\left(1.84 \log \mathrm{cd} \times \mathrm{s} / \mathrm{m}^{2}\right)$ to dimmest $(-3.56$ $\log \mathrm{cd} \times \mathrm{s} / \mathrm{m}^{2}$ ) background light intensity. Intriguingly, we observed

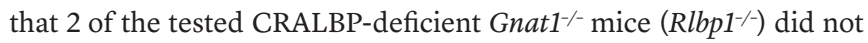
respond normally to the moving bar in the test and instead rotated their head in the direction opposite to the moving bar. Therefore, we excluded the results from these 2 mice from our analysis.

Electrophysiology. In vivo ERG recordings were performed with a commercial LKC system (LKC Technologies) as previously described (6). The dark-adapted animals were anesthetized with ketamine $(100 \mathrm{mg} / \mathrm{kg})$ and xylazine $(20 \mathrm{mg} / \mathrm{kg})$, and their pupils were dilated with atropine sulfate eye drops. A mouse was placed onto a $37^{\circ} \mathrm{C}$ heating pad, and electrodes were connected to its cornea to amplify electrical signals generated by the retina. A reference electrode was connected to beneath the skin at the scalp between the eyes. The animal was allowed to stabilize in darkness for 15 minutes before the recordings were started. Test flashes of increasing intensity (530-nm LED up to a $25 \mathrm{~cd} \times \mathrm{s} / \mathrm{m}^{2}$ limit and white Xenon flash for higher intensities) were delivered by the Ganzfeld sphere, and the ERG signals were recorded to obtain the intensity-response curves. To test the dark-adaptation kinetics, bright-green LED light $(520 \mathrm{~nm})$ was used to bleach an estimated $90 \%$ of the photopigments in 30 seconds. Then the cone sensitivity was recorded at preset time intervals until 52 minutes after photobleaching. The cone b-wave flash sensitivity was normalized to the pre-bleaching value to determine the sensitivity recovery curve. For exogenous chromophore application experiments, $300 \mu \mathrm{g}$ 9-cis retinal was dissolved in $200 \mu \mathrm{l} \mathrm{NaCl} / \mathrm{BSA}$ solution (with $10 \%$ ethanol) and administered by i.p. injection. The treated animals were dark adapted overnight prior to in vivo ERG recordings.

Transretinal recordings were performed with isolated mouse retina as previously described $(49,50)$. The isolated retina was carefully mounted on a custom-made chamber for transretinal voltage recordings (51). The retina was perfused with Locke's solution bubbled with $\mathrm{O}_{2}$ and $\mathrm{CO}_{2}$ and supplemented with $30 \mu \mathrm{M}$ DL-AP4 to block synaptic transmission. After setting up the recording chamber, the retina was allowed to stabilize for 15 minutes before any recording was done. The responses of cones to 1-ms-long 505-nm LED-generated test flashes of various intensities were amplified and recorded on a desktop computer with pClamp 10 software (Molecular Dynamics). Dim flash analysis was performed with amplitude responses below $30 \%$ of the maximal amplitude for each retina (52). For dark-adaptation tests, preprogrammed protocols were used to precisely monitor the fast recovery of cone sensitivity during the first 12 minutes following an estimated $90 \%$ photobleaching of the visual pigments. The cone sensitivity was normalized to the pre-bleaching level to generate the sensitivity recovery curve. For exogenous chromophore application experiments, $300 \mu \mathrm{g}$ 11 -cis retinal was dissolved in $4 \mathrm{ml} 0.01 \%$ ethanol Locke's solution, and an isolated retina was incubated with 0.5 to $1 \mathrm{ml}$ of that solution for 1 hour in darkness. Cone responses from the treated retinae were 
then obtained using transretinal recordings. For all recordings, the sensitivity, $\mathrm{S}_{\mathrm{f}}$, was estimated from the ratio of the amplitude, $\mathrm{r}$, of a flash response from the linear range $\left(<30 \%\right.$ of the saturated response, $\left.r_{\max }\right)$ and the corresponding flash intensity.

Frozen sections. Eyes were fixed in $4 \%$ paraformaldehyde in PBS for 2 hours at room temperature, rinsed with PBS, and then cryoprotected in $30 \%$ sucrose. The lens was removed prior to embedding in Tissue-Tek OCT compound (Sakura). Frozen blocks were cryosectioned at a thickness of 12 to $14 \mu \mathrm{m}$. For immunohistochemical staining, sections were blocked for approximately 1 hour at room temperature, followed by overnight incubation at $4^{\circ} \mathrm{C}$ with primary antibody, except for anti-CRALBP, which was incubated for 2 hours at room temperature. The following primary antibodies were used: rabbit anti-red/green cone opsin (AB5405; EMD Millipore) at 1:600; rabbit anti-blue cone opsin (AB5407; EMD Millipore) at 1:200; and rabbit anti-CRALBP (UW55 polyclonal antibody isolated from rabbits immunized with human recombinant CRALBP; a gift of John Saari, University of Washington; refs. 22, 53) at 1:200. The following fluorescently labeled secondary antibodies were used, respectively: Alexa Fluor 555 donkey anti-rabbit (A-31572; Molecular Probes) at 1:800; Alexa Fluor 488 donkey anti-rabbit (A-21206; Molecular Probes) at 1:500; and Alexa Fluor 555 donkey anti-rabbit (A-31572; Molecular Probes) at 1:800. Secondary antibodies were applied for 30 minutes at room temperature, followed by DAPI staining, application of VECTASHIELD (Vector Labs), and coverslipping. The following blocking solutions were used: $0.1 \%$ Triton X-100 and 5\% normal donkey serum in PBS for staining of opsins, and 0.5\% Triton $\mathrm{X}-100$ and 2\% normal donkey serum in PBS for staining of CRALBP. Primary and secondary antibodies were diluted in blocking solution. Slides were stored at $-20^{\circ} \mathrm{C}$ until imaging. Images were taken as multi-plane captures using an Olympus BX61WI microscope and a Hamamatsu ORCA-AG CCD camera and processed with MetaMorph software (Molecular Devices) and Adobe Photoshop, except for Figure 6, E and F, which were taken as single-plane captures using an Olympus BX51 microscope and an Olympus DP70 camera and processed with SlideBook software (Intelligent Imaging Innovations) and Adobe Photoshop.

Whole-mount immunostaining. Retinae were dissected in PBS with the lens intact, fixed for 30 minutes at room temperature with $4 \%$ paraformaldehyde in PBS, and rinsed with PBS prior to removal of the lens. Retinae were blocked for approximately 1 hour at room temperature, followed by overnight incubation at $4^{\circ} \mathrm{C}$ with the primary antibody. The following primary antibodies were used: rabbit antired/green cone opsin (AB5405; EMD Millipore) at 1:500 and goat anti-blue opsin (sc-14363; Santa Cruz Biotechnology Inc.) at 1:500. The following fluorescently labeled secondary antibodies were used, respectively: Alexa Fluor 555 donkey anti-rabbit (A-21206; Molecular Probes) at 1:800 and Alexa Fluor 488 donkey anti-goat (A-11055; Molecular Probes) at 1:800. Secondary antibodies were applied for 30 minutes at room temperature. A blocking solution consisting of $0.5 \%$ Triton $\mathrm{X}-100$ and $2 \%$ normal donkey serum in PBS was used. Primary and secondary antibodies were diluted in blocking solution. Retinae were stored light-protected in PBS at $4^{\circ} \mathrm{C}$ until imaging, whereupon they were whole mounted with glass shards at the corners of the slide and coverslipped.

Cone quantification. Whole-mounted retinae were imaged as single-plane captures using a $\times 10$ objective lens with an Olympus BX51 microscope and an Olympus DP70 camera. Image files were annotated in Adobe Photoshop with dots to mark opsin staining. The dots were quantified using the "Analyze Particles" feature in ImageJ software (NIH). Quantification was conducted in $170 \mu \mathrm{m} \times 170 \mu \mathrm{m}$ fields located in the dorsal, nasal, temporal, and ventral quadrants.

$A A V$ vector preparation and injection. Four types of AAV vectors were used for the injection: shH10-scCAG-RLBP1 (expressing CRALBP in Müller cells, Müller-CRALBP); 7m8-scVMD2-RLBP1 (expressing CRALBP in RPE cells, RPE-CRALBP); shH10-scCAG-GFP (expressing GFP in Müller cells, GFP control); and 7m8-scVMD2-GFP (expressing GFP in RPE cells, GFP control). The Müller glia-specific viruses (shH10-scCAG-RLBP1/GFP) were constructed using a Müller glia-specific AAV serotype shH10 and the ubiquitous synthetic $C A G$ promoter (54). The RPE-specific viruses (7m8-scVMD2-RLBP1/GFP) were built using a pan-retinally expressing AAV serotype $7 \mathrm{~m} 8$ and the RPE-specific promoter VMD2 $(55,56)$. To generate the scCAG-RLBP1 viral transfer plasmid, RLBP1 was reverse transcribed from purified WT mouse retina total mRNA. Then the GFP ORF of a self-complementary AAV vector expressing GFP under CAG promoter control was replaced with the Rlbp1 cDNA using restriction enzymes. This plasmid was then further processed to generate the scVMD2-RLBP1 transfer plasmid through replacement of the $C A G$ promoter with the PCR-purified VMD2 promoter. The control scCAG-GFP and scVMD2-GFP viral plasmids were created using the same process without the replacement of the GFP ORF. For the generation of each virus, 293 T cells at $80 \%$ confluence were cotransfected with the appropriate transfer plasmid, pHelper plasmid, and the AAV rep/cap plasmid (shH10 or $7 \mathrm{~m} 8$ ) at a molar ratio of 1:1:1. At 72 hours after transfection, cells were collected, pelleted, resuspended in lysis buffer, freezethawed, and then treated with Benzonase. Cell debris was removed by centrifugation, and the supernatant was loaded onto an iodixanol gradient and subjected to ultracentrifugation. The $40 \%$ virus-containing iodixanol fraction was removed from the gradient, and the iodixanol was replaced via buffer exchange using Amicon Ultra-15 Centrifugal units in PBS. Titers were determined by quantitative PCR relative to a standard curve (57). An aliquot of 1 to $1.5 \mu \mathrm{l}$ of the virus was injected into the vitreous of anesthetized 4-week-old mice using a Hamilton syringe. Animals were harvested $4-5$ weeks after the injection for cone electrophysiology experiments and 8 weeks after the injection for immunohistochemistry.

Pupillary light reflex. All mice were awake and manually restrained while a 480-nm LED light was directed to 1 eye (the left eye). The light stimulus lasted for 30 seconds, after which the mouse was returned to its cage to dark adapt until the next light stimulus. Individual frames of the movie were taken from VLC Media Player. The images were analyzed in ImageJ. A comparison was made of the pupil area in darkness and after 30 seconds of light exposure to generate a ratio. For doseresponse curves, the data were fitted with a variable-slope sigmoidal dose-response curve, with the top constrained to 1.0 and the bottom constrained between 0 and 0.1 .

Statistics. Unless otherwise noted, a 2-tailed unpaired Student's $t$ test was used to test for the significance of differences in the mean values of 2 sample groups. A $P$ value of less than 0.05 was considered statistically significant.

Study approval. The care, maintenance, and treatment of the animals in this study followed protocols approved by the Washington University Animal Studies Committee. 


\section{Acknowledgments}

This work was supported by NIH grants EY019312 and EY021126 (to V.J. Kefalov); EY18826, HG006790, and HG006346 (to J.C. Corbo); GM076430-09 (to S. Hattar); 5T32EY013360-15 (to S.Q. Shen); and EY002687 to the Department of Ophthalmology and Visual Sciences at Washington University; and by grants from The Foundation Fighting Blindness (to J.G. Flannery); the Human Frontier Science Program (to J.C. Corbo); and Research to Prevent Blindness. We thank John Saari of the University of Washington for the CRALBP-deficient mice and the CRALBP antibody; the National Eye Institute and Rosalie Crouch of the Medical Uni- versity of South Carolina for the 11-cis retinal; and Michael Casey of the Transgenic Core of the Department of Ophthalmology at Washington University for genotyping our mice for the $r d 8$ mutation. We also thank Alexander Kolesnikov and Frans Vinberg of the Kefalov Laboratory, Carter Cornwall of Boston University, and Huixin Xu of Harvard University for comments on the manuscript.

Address correspondence to: Vladimir J. Kefalov, Department of Ophthalmology and Visual Sciences, Washington University in St. Louis, 660 South Euclid Avenue, St. Louis, Missouri 63110, USA. Phone: 314.362.4376; E-mail: kefalov@wustl.edu.
1. Saari JC. Vitamin A metabolism in rod and cone visual cycles. Annu Rev Nutr. 2012;32:125-145.

2. Wang J-S, Kefalov VJ. The cone-specific visual cycle. Prog Retin Eye Res. 2011;30(2):115-128.

3. Mata NL, Radu RA, Clemmons RS, Travis GH. Isomerization and oxidation of vitamin a in cone-dominant retinas: a novel pathway for visual-pigment regeneration in daylight. Neuron . 2002;36(1):69-80.

4. Wang J-S, Estevez ME, Cornwall MC, Kefalov VJ. Intra-retinal visual cycle required for rapid and complete cone dark adaptation. Nat Neurosci. 2009;12(3):295-302.

5. Wang J-S, Kefalov VJ. An alternative pathway mediates the mouse and human cone visual cycle. Curr Biol. 2009;19(19):1665-1669.

6. Kolesnikov AV, Tang PH, Parker RO, Crouch RK, Kefalov VJ. The Mammalian cone visual cycle promotes rapid $\mathrm{m} / \mathrm{l}$-cone pigment regeneration independently of the interphotoreceptor retinoid-binding protein. J Neurosci. 2011;31(21):7900-7909.

7. Kaylor JJ, et al. Identification of DES1 as a vitamin A isomerase in Müller glial cells of the retina. Nat Chem Biol. 2013;9(1):30-36.

8. Kaylor JJ, et al. Identification of the 11-cis-specific retinyl-ester synthase in retinal Müller cells as multifunctional O-acyltransferase (MFAT). Proc Natl Acad Sci U S A. 2014;111(20):7302-7307.

9. Liu T, Jenwitheesuk E, Teller DC, Samudrala R. Structural insights into the cellular retinaldehyde-binding protein (CRALBP). Proteins. 2005;61(2):412-422.

10. Fleisch VC, Schonthaler HB, von Lintig J, Neuhauss SCF. Subfunctionalization of a retinoid-binding protein provides evidence for two parallel visual cycles in the cone-dominant zebrafish retina. J Neurosci. 2008;28(33):8208-8216.

11. Collery R, et al. Duplication and divergence of zebrafish CRALBP genes uncovers novel role for RPE- and Muller-CRALBP in cone vision. Invest Ophthalmol Vis Sci. 2008;49(9):3812-3820.

12. Babino D, Perkins BD, Kindermann A, Oberhauser $\mathrm{V}$, von Lintig J. The role of 11-cis-retinyl esters in vertebrate cone vision. FASEB J. 2015;29(1):216-226

13. Maw MA, et al. Mutation of the gene encoding cellular retinaldehyde-binding protein in autosomal recessive retinitis pigmentosa. Nat Genet. 1997;17(2):198-200.

14. Burstedt MS, Sandgren O, Holmgren G, Forsman-Semb K. Bothnia dystrophy caused by mutations in the cellular retinaldehyde-binding protein gene (RLBP1) on chromosome 15q26. Invest Ophthalmol Vis Sci. 1999;40(5):995-1000.

15. Burstedt MS, et al. Ocular phenotype of bothnia dystrophy, an autosomal recessive retinitis pigmentosa associated with an $\mathrm{R} 234 \mathrm{~W}$ mutation in the RLBP1 gene. Arch Ophthalmol. 2001;119(2):260-267.

16. Burstedt MSI, Sandgren O, Golovleva I, Wachtmeister L. Retinal function in Bothnia dystrophy. An electrophysiological study. Vision Res. 2003;43(24):2559-2571.

17. Morimura H, Berson EL, Dryja TP. Recessive mutations in the RLBP1 gene encoding cellular retinaldehyde-binding protein in a form of retinitis punctata albescens. Invest Ophthalmol Vis Sci. 1999;40(5):1000-1004.

18. Naz S, et al. Mutations in RLBP1 associated with fundus albipunctatus in consanguineous Pakistani families. Br JOphthalmol. 2011;95(7):1019-1024.

19. Katsanis N, et al. Fundus albipunctatus and retinitis punctata albescens in a pedigree with an R150Q mutation in RLBP1. Clin Genet. 2001;59(6):424-429.

20. Eichers ER, et al. Newfoundland rod-cone dystrophy, an early-onset retinal dystrophy, is caused by splice-junction mutations in RLBP1. Am J Hum Genet. 2002;70(4):955-964.

21. Thompson DA, Gal A. Vitamin A metabolism in the retinal pigment epithelium: genes, mutations, and diseases. Prog Retin Eye Res. 2003;22(5):683-703.

22. Saari JC, et al. Visual cycle impairment in cellular retinaldehyde binding protein (CRALBP) knockout mice results in delayed dark adaptation. $\mathrm{Neu}$ ron. 2001;29(3):739-748.

23. Calvert PD, et al. Phototransduction in transgenic mice after targeted deletion of the rod transducin alpha -subunit. Proc Natl Acad Sci U S A. 2000;97(25):13913-13918.

24. Nikonov SS, Kholodenko R, Lem J, Pugh EN. Physiological features of the S- and M-cone photoreceptors of wild-type mice from single-cell recordings. J Gen Physiol. 2006;127(4):359-374.

25. Keller C, Grimm C, Wenzel A, Hafezi F, Remé C. Protective effect of halothane anesthesia on retinal light damage: inhibition of metabolic rhodopsin regeneration. Invest Ophthalmol Vis Sci. 2001;42(2):476-480.

26. Ablonczy Z, et al. 11-cis-retinal reduces constitutive opsin phosphorylation and improves quantum catch in retinoid-deficient mouse rod photoreceptors. J Biol Chem. 2002;277(43):40491-40498.

27. Rohrer B, et al. Correlation of regenerable opsin with rod ERG signal in Rpe $65^{-/-}$mice during development and aging. Invest Ophthalmol Vis Sci. 2003;44(1):310-315.

28. Dunn FA, Della Santina L, Parker ED, Wong ROL. Sensory experience shapes the development of the visual system's first synapse. Neuron. 2013;80(5):1159-1166.

29. Hattar S, et al. Melanopsin and rod-cone photoreceptive systems account for all major accessory visual functions in mice. Nature. 2003;424(6944):76-81.

30. Wong K. Intrinsically photosensitive retinal ganglion cells (ipRGCs) depend on the retinal pigment epithelium (RPE) to regenerate melanopsin [abstract]. Invest Ophthalmol Vis Sci. 2013;54:400.

31. Xue T, et al. Melanopsin signalling in mammalian iris and retina. Nature. 2011;479(7371):67-73.

32. Lucas RJ, et al. Diminished pupillary light reflex at high irradiances in melanopsin-knockout mice. Science. 2003;299(5604):245-247.

33. Tu DC, et al. Inner retinal photoreception independent of the visual retinoid cycle. Proc Natl Acad Sci U S A. 2006;103(27):10426-10431.

34. Goldstein EB. Cone pigment regeneration in the isolated frog retina. Vision Res. 1970;10(10):1065-1068.

35. Muniz A, et al. Evidence for two retinoid cycles in the cone-dominated chicken eye. Biochemistry. 2009;48(29):6854-6863.

36. Saari JC, Crabb JW. Focus on molecules: cellular retinaldehyde-binding protein (CRALBP). Exp Eye Res. 2005;81(3):245-246.

37. Nawrot M, et al. Cellular retinaldehyde-binding protein interacts with ERM-binding phosphoprotein 50 in retinal pigment epithelium. Invest Ophthalmol Vis Sci. 2004;45(2):393-401.

38. Bonilha VL, et al. Support for a proposed retinoid-processing protein complex in apical retinal pigment epithelium. Exp Eye Res. 2004;79(3):419-422.

39. Jin $\mathrm{M}$, et al. The role of interphotoreceptor retinoid-binding protein on the translocation of visual retinoids and function of cone photoreceptors. JNeurosci. 2009;29(5):1486-1495.

40. Parker RO, Fan J, Nickerson JM, Liou GI, Crouch RK. Normal cone function requires the interphotoreceptor retinoid binding protein. J Neurosci. 2009;29(14):4616-4621.

41. Redmond TM, et al. Rpe65 is necessary for production of 11-cis-vitamin $\mathrm{A}$ in the retinal visual cycle. Nat Genet. 1998;20(4):344-351.

42. Znoiko SL, et al. Downregulation of cone-specific 
gene expression and degeneration of cone photoreceptors in the Rpe $65^{-/}$mouse at early ages. Invest Ophthalmol Vis Sci. 2005;46(4):1473-1479.

43. Jacobson SG, et al. Human cone photoreceptor dependence on RPE65 isomerase. Proc Natl Acad Sci U S A. 2007;104(38):15123-15128.

44. Cottet $S$, et al. Biological characterization of gene response in Rpe65-/- mouse model of Leber's congenital amaurosis during progression of the disease. FASEB J. 2006;20(12):2036-2049.

45. Insinna C, et al. An S-opsin knock-in mouse (F81Y) reveals a role for the native ligand 11-cisretinal in cone opsin biosynthesis. J Neurosci. 2012;32(23):8094-8104.

46. Zhang H, et al. Trafficking of membrane-associated proteins to cone photoreceptor outer segments requires the chromophore 11-cis-retinal. J Neurosci. 2008;28(15):4008-4014.

47. Cunea A, Powner MB, Jeffery G. Death by color: differential cone loss in the aging mouse retina.
Neurobiol. 2014;35(11):2584-2591.

48. Mattapallil MJ, et al. The Rd8 mutation of the Crb1 gene is present in vendor lines of C57BL/6N mice and embryonic stem cells, and confounds ocular induced mutant phenotypes. Invest $\mathrm{Oph}$ thalmol Vis Sci. 2012;53(6):2921-2927.

49. Sundermeier TR, et al. DICER1 is essential for survival of postmitotic rod photoreceptor cells in mice. FASEB J. 2014;28(8):3780-3791.

50. Sundermeier TR, et al. R9AP overexpression alters phototransduction kinetics in iCre75 mice. Invest Ophthalmol Vis Sci. 2014;55(3):1339-1347.

51. Vinberg F, Kolesnikov AV, Kefalov VJ. Ex vivo ERG analysis of photoreceptors using an in vivo ERG system. Vision Res. 2014;101:108-117.

52. Pugh EN, Lamb TD. Amplification and kinetics of the activation steps in phototransduction. Biochim Biophys Acta. 1993;1141(2-3):111-149.

53. Saari JC, Nawrot M, Stenkamp RE, Teller DC, Garwin GG. Release of 11-cis-retinal from cellu- lar retinaldehyde-binding protein by acidic lipids. Mol Vis. 2009;15:844-854.

54. Klimczak RR, Koerber JT, Dalkara D, Flannery JG, Schaffer DV. A novel adeno-associated viral variant for efficient and selective intravitreal transduction of rat Müller cells. PLoS One. 2009;4(10):e7467.

55. Dalkara D, et al. In vivo-directed evolution of a new adeno-associated virus for therapeutic outer retinal gene delivery from the vitreous. Sci Transl Med. 2013;5(189):189ra76.

56. Esumi N, Oshima Y, Li Y, Campochiaro PA, Zack DJ. Analysis of the VMD2 promoter and implication of E-box binding factors in its regulation. J Biol Chem. 2004;279(18):19064-19073.

57. Aurnhammer C, et al. Universal real-time PCR for the detection and quantification of adenoassociated virus serotype 2-derived inverted terminal repeat sequences. Hum Gene Ther. 2012;23(1):18-28. 